\title{
AUTOMATED LOWER BOUND ANALYSIS OF CONCRETE SLABS
}

\author{
Chris BURGOYNE ${ }^{1}$ \\ Andy SMITH ${ }^{2}$ \\ 1 Department of Engineering, University of Cambridge, UK \\ 2 Steel Construction Institute, UK
}

Keywords: Concrete Slabs, Lower Bound Method, Hillerborg Strip, Structural Analysis

\section{INTRODUCTION}

The problems caused by using different methods for the design of concrete slabs, and their subsequent reanalysis for assessment, were highlighted in an earlier paper [1]. If the original calculations have been lost it is common for structures to be condemned because the checker does not know the original designer's logic.

In many cases the design was carried out by hand calculation, often using the Hillerborg strip method [2, 3] or code rules that were derived from it. That method assumes a distribution of load between two sets of strips, usually orthogonal, for which the reinforcement can then be designed independently. The engineer's choice of load distribution is arbitrary, and the resulting moments may differ from those in the real structure, but the Lower Bound Theorem of Plasticity [4] means that the structure will be safe. As long as the engineer has provided sufficient reinforcement for the moments that have been calculated, and has complied with normal code rules to ensure ductility, the difference between the actual and assumed moments does not matter. The worst that could happen is that the structure would crack prematurely in the under-strength direction, but it would be strong enough in the other direction and the structure would be safe.

The problem is that the checker does not know how the original designer chose to distribute the loads, or even what type of analysis was performed. Modern finite element packages are now so easy to use, at least in their linear elastic incarnations, and give such pretty pictures as output, that the analyst is tempted to believe that the results are precise. Many structures are being wrongly condemned. May \& Mann [5] have recently shown that a fully nonlinear finite element analysis does allow the post-cracking and post-yield behaviour of the slab to be followed, but the use of such programs requires expert knowledge and is not undertaken in routine assessment.

There have been attempts to automate the use of the Plasticity Bound Theorems for design and analysis. Middleton [6] has developed a technique to automate the Yield Line Method, primarily aimed at the assessment of bridge decks. He uses a library of possible failure mechanisms whose parameters are altered to get the Lowest Upper Bound. He has conducted many tests to show that, despite being an Upper Bound, his predictions are sufficiently close to the true collapse load that they can be used as the basis for an assessment of structural safety. It has also been observed that membrane forces are frequently developed which cause the final collapse load to be above the upper bound prediction. Nevertheless fears are still expressed that the results are on the unsafe side, by definition.

The Hillerborg Strip method has been automated before by O'Dwyer and O'Brien [7], using a technique that differs from the one presented here. It will be shown below that both techniques are satisfactory as a design tools, but the Hillerborg approach itself has to be modified for use as an analysis tool.

What is needed is a method that can be used to automate the Lower Bound Method for use in analysis, so that the Highest Lower Bound can be compared with the Lowest Upper Bound. Even though neither method gives the true collapse load, if the two bounds differ by only a small amount the checker can have confidence in the judgement that is made of the slab's capacity. This paper presents a method to address that problem.

\subsection{Sign Convention}

Figure 1 shows the sign convention used for moments, which is consistent with that used for most reinforced concrete slab work. As defined, positive moments relate to hogging moments and are thus resisted by the top steel in a slab, and $M_{x}$ refers to moments resisted by steel running in the $x$-direction and not to moments about the $x$-axis. In most cases, rigour of sign convention is not required, and reference will be made to sagging and hogging moments, and to longitudinal strips (which run in the $x$ - 
direction) and transverse strips (which run in $y$-direction). The methods described here can easily be adapted to any other self-consistent sign convention.

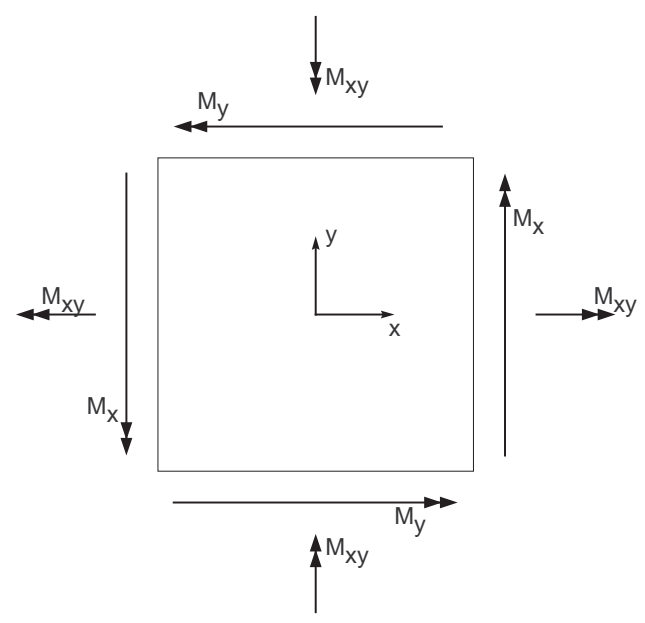

Fig 1. Sign convention for moments

\section{HILLERBORG STRIP METHOD}

The standard Hillerborg Strip Method assumes that the slab is made up of two sets of intersecting strips; they will be taken here to be orthogonal but the extension to non-orthogonal strips is straightforward. The basic equilibrium equation for flexure of a plate is

$$
\frac{\partial^{2} M_{x}}{\partial x^{2}}+\frac{\partial^{2} M_{x y}}{\partial x . \partial y}+\frac{\partial^{2} M_{y}}{\partial y^{2}}=q
$$

Hillerborg chose to make $M_{x y}$ zero, which allowed a simpler equilibrium system to be set up in which the applied load $q$ could be shared between the two strips with $\alpha q$ applied to one strip and (1$\alpha) q$ applied to the other strip. Hillerborg was almost certainly aware that resisting $M_{x y}$ moments is also more costly in reinforcement. The equilibrium equations for the two strips could then be separated.

$$
\begin{aligned}
& \frac{\partial^{2} M_{x}}{\partial x^{2}}=\alpha q \\
& \frac{\partial^{2} M_{y}}{\partial y^{2}}=(1-\alpha) q
\end{aligned}
$$

The reinforcement in each strip can then be designed on the basis of beam theory; if the strips are designed in accordance with normal code rules the strips will be under-reinforced and will fail in flexure before they fail in shear. The Lower Bound Theorem of Plasticity then applies and the structure will be safe whatever value of $\alpha$ has been chosen. In practice, codes often specify how the load is to be distributed, using "column strips" and "middle strips", but these requirements are designed to prevent early cracking of the slab and are not required for safety.

As originally proposed, the method cannot deal with patch loads, since many strips will be unloaded, but a set of self-equilibrating forces $p$ can be introduced between the two sets of strips whose total external effect is zero. This would leave the designer with two parameters $\alpha$ and $p$ that can be chosen at will, but without any loss of generality, the problem can be simplified by setting $\alpha$ to unity (thus applying all the load to the strips in the $\mathrm{x}$-direction) and using $p$ to specify how the load is transferred within the slab. 


$$
\begin{aligned}
& \frac{\partial^{2} M_{x}}{\partial x^{2}}=q+p \\
& \frac{\partial^{2} M_{y}}{\partial y^{2}}=-p
\end{aligned}
$$

As described above, the method is suitable for design. The problem now is to reverse the usual process so that an existing structure can be analysed. Instead of choosing $p$, and then calculating the required moment capacities $m_{x}$ and $m_{y}$, a method is needed to find the $p$ values for a slab with a known moment capacity. This is much more complex; the design problem leads directly to a solution, whereas the inverse problem requires searching for optimal values of $p$, and there are many more of these than there are pattern parameters in the normal yield line analysis.

\section{THE METHOD}

Consider a rectangular slab divided into orthogonal regions, as shown in Fig. 2, which defines two sets of strips. The applied load and moment capacities are assumed to be constant within each region, and the boundary conditions for the strips are constant for each set of strips. The moments in the strips can be completely defined by the values of $p_{i}$ assigned to each rectangular region.

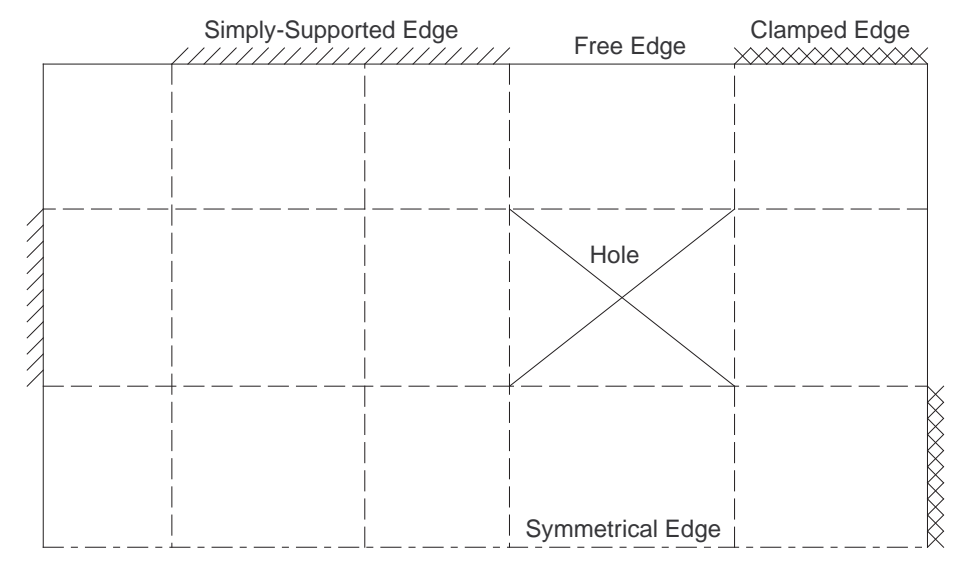

Fig 2. General slab showing notation for boundary conditions.

Moment capacities and loads are constant within each rectangular region

The objective is to determine a distributed load, $Q_{\max }$ (dimensions $\mathrm{FL}^{-2}$ ) that can be multiplied by the dimensionless load distribution, $q_{i}$, which describes how the load is applied to the slab, to give the predicted maximum loading for the structure, $Q_{\max } q_{i}$. The loads applied to the longitudinal strips are thus $Q_{\max }\left(q_{i}+p_{i}\right)$ and those on the transverse strips $-Q_{\max } p_{i}$. The values of $q_{i}$ are known but the $p_{i}$ have to be determined subject to the condition that the moment capacity in each strip, in either hogging or sagging bending, cannot be exceeded.

Allowance has to be made for the support conditions for a strip. A general strip (Fig. 3) has up to four end reactions or moments; depending on the type of support some of these may be zero or there will be additional equilibrium equations that have to be satisfied.

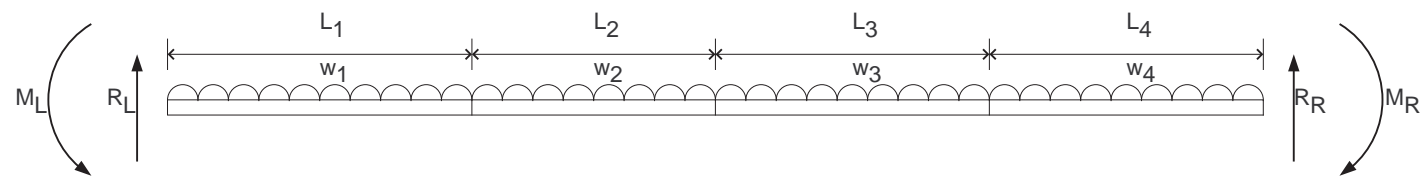

Fig 3. Notation for loading on strip. 
For a strip that is fixed at both ends, $M_{L}$ and $M_{R}$ can take any values and it will still be possible to determine $R_{L}$ and $R_{R}$ using the two equations of overall equilibrium for the strip. However, if the right hand end is free, the left-hand edge moment $M_{L}$ will have a fixed relationship to the applied loads $w_{i}$ in order for overall equilibrium to be satisfied.

For a strip that is free at both ends the applied loads themselves will have to satisfy both vertical and moment equilibrium so that all the end reactions and moments are zero. In both cases the edge moment for a free end will be set to zero.

By considering every strip that makes up the complete slab and replacing $w_{i}$ with the loads given by Eq. (3) a set of simultaneous equations can be derived that defines an equilibrium system for the slab. This can be expressed in matrix form as:

$$
A p^{*}=b
$$

$\mathbf{p}^{*}$ is a column vector of internal loads, $p_{i}$, and the non-zero edge moments; $\mathbf{A}$ and $\mathbf{b}$ are the matrix and column vector that contain the terms from the simultaneous equations described above. A holds the values that relate to the entries in the $\mathbf{p}^{*}$ vector and $\mathbf{b}$ holds the values that relate to the applied loads, $q_{i}$. Equation (4) defines the equilibrium of the system, and by using values for $\mathbf{p}^{*}$ that satisfy this equation the support conditions will always be satisfied.

The shape of the matrix $\mathbf{A}$ depends on the degree of statical indeterminacy. If the system is determinate the matrix will be square and the moment distribution is fixed. Normally $\mathbf{A}$ will have more columns than rows, reflecting statical indeterminacy, and the solution will take the form:

$$
\mathbf{p}^{*}=\mathrm{e}+\mathrm{S} \lambda
$$

Here $\mathbf{e}$ is an equilibrium state of the system while $\mathbf{S}$ is a matrix composed of a series of column vectors, each of which is a state of self-stress: $\lambda$ is a column vector of variables which add differing amounts of the states of self-stress to the equilibrium state. Thus the objective is to choose the values of $\lambda$ that maximise $Q_{\max }$. This reduces the number of variables that have to be optimised later, significantly speeding up the process. The method can be extended to deal with columns, holes and lines of symmetry, but details are not given here

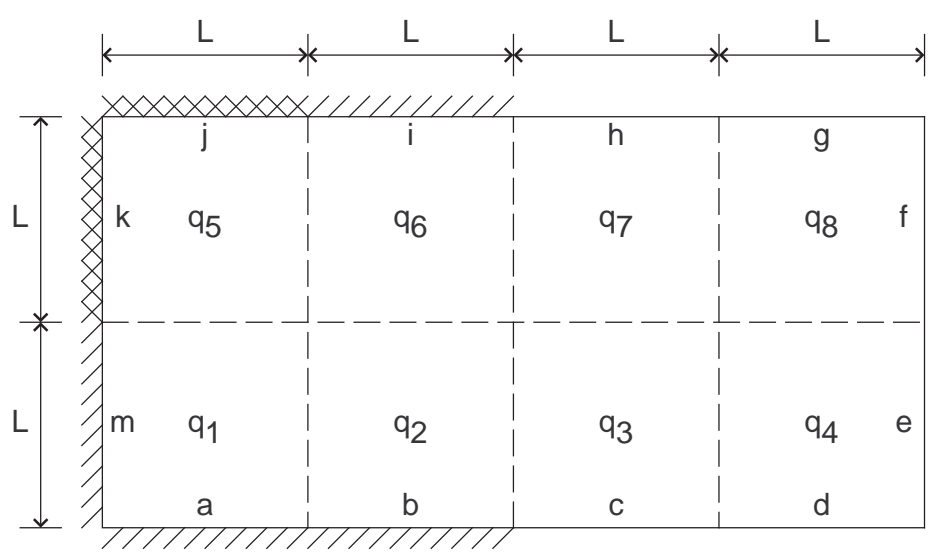

Fig 4. Example to demonstrate equilibrium.

The method can most easily be demonstrated by means of an example. The slab shown in Fig. 4 is divided into 8 regions, each of which can be loaded independently by choosing values of $q_{i}$. There are simple supports at edges $\mathrm{a}, \mathrm{b}, \mathrm{i}$, and $\mathrm{m}$, and clamped supports at edges $\mathrm{j}$ and $\mathrm{k}$. In addition to the eight internal forces $\left(p_{1}\right.$ to $\left.p_{8}\right)$ there will be two edge moments $\left(M_{j}\right.$ and $\left.M_{k}\right)$, so the vector $\mathbf{p}^{*}$ has 10 components. Once these are determined, the support reactions and moments in all strips can be calculated. The example is slightly artificial but it shows all aspects of the way the method has been implemented.

To find $\mathbf{p}^{*}$, the matrices $\mathbf{A}$ and $\mathbf{b}$ have to be found. The relationship between the variables needs to be determined so that the edge conditions are satisfied. Consider first the longitudinal strip m-e. To establish equilibrium for this strip the moment applied by the four loads, $q_{i}+p_{i}$, about edge m must be zero, giving: 


$$
\left(q_{1}+p_{1}\right) \frac{L^{2}}{2}+\left(q_{2}+p_{2}\right) \frac{3 L^{2}}{2}+\left(q_{3}+p_{3}\right) \frac{5 L^{2}}{2}+\left(q_{4}+p_{4}\right) \frac{7 L^{2}}{2}=0
$$

Moment equilibrium of strip $k-f$ gives a similar equation:

$$
\left(q_{5}+p_{5}\right) \frac{L^{2}}{2}+\left(q_{6}+p_{6}\right) \frac{3 L^{2}}{2}+\left(q_{7}+p_{7}\right) \frac{5 L^{2}}{2}+\left(q_{8}+p_{8}\right) \frac{7 L^{2}}{2}=M_{k}
$$

Transverse strips a-j and b-i will be in equilibrium for any value of the applied loads and edge moments since the reaction forces at both ends of both strips can be adjusted, so these need not be taken into account at this stage. For strips $c-h$ and d-g the applied loads, $-p_{i}$, will need to satisfy both force and moment equilibrium. Taking moments about $\mathrm{c}$ and $\mathrm{d}$ gives:

$$
\begin{gathered}
\sum V:-p_{3} L+-p_{7} L=0 \\
M: \frac{-p_{3} L^{2}}{2}+\frac{-3 p_{7} L^{2}}{2}=0 \\
\sum V:-p_{4} L+-p_{8} L=0 \\
M: \frac{-p_{4} L^{2}}{2}+\frac{-3 p_{8} L^{2}}{2}=0
\end{gathered}
$$

There are thus 6 equations defining 10 variables:

$$
\left[\begin{array}{cccccccccc}
\frac{L^{2}}{2} & \frac{3 L^{2}}{2} & \frac{5 L^{2}}{2} & \frac{7 L^{2}}{2} & 0 & 0 & 0 & 0 & 0 & 0 \\
0 & 0 & 0 & 0 & \frac{L^{2}}{2} & \frac{3 L^{2}}{2} & \frac{5 L^{2}}{2} & \frac{7 L^{2}}{2} & 0 & -1 \\
0 & 0 & -L & 0 & 0 & 0 & -L & 0 & 0 & 0 \\
0 & 0 & \frac{-L^{2}}{2} & 0 & 0 & 0 & \frac{-3 L^{2}}{2} & 0 & 0 & 0 \\
0 & 0 & 0 & -L & 0 & 0 & 0 & -L & 0 & 0 \\
0 & 0 & 0 & \frac{-L^{2}}{2} & 0 & 0 & 0 & \frac{-3 L^{2}}{2} & 0 & 0
\end{array}\right]\left[\begin{array}{c}
p_{1} \\
p_{2} \\
p_{3} \\
p_{4} \\
p_{5} \\
p_{6} \\
p_{7} \\
p_{8} \\
M_{j} \\
M_{k}
\end{array}\right]=\left[\begin{array}{c}
\frac{-\left(q_{1}+3 q_{2}+5 q_{3}+7 q_{4}\right) L^{2}}{2} \\
\frac{-\left(q_{5}+3 q_{6}+5 q_{7}+7 q_{8}\right) L^{2}}{2} \\
0 \\
0 \\
0 \\
0
\end{array}\right]
$$

All the loading terms are on the right hand side of this equation, which can be rearranged by Gaussian elimination; pivoting reorders the variables. For a uniform load over the entire slab, $q_{1}=q_{2}=$ $\ldots=q_{8}=q$, and with $L=1 \mathrm{~m}$, equation (12) becomes: 


$\left[\begin{array}{cccccccccc}1 & 0.068 & 0.593 & 0.356 & 0 & 0 & 0.119 & 0 & 0 & -0.237 \\ 0 & 1 & -0.047 & -0.028 & 0.690 & 0.414 & -0.009 & 0.138 & 0 & 0.019 \\ 0 & 0 & 1 & 0.175 & 0.399 & 0.070 & 0.058 & 0.023 & 0 & -0.117 \\ 0 & 0 & 0 & 1 & -0.005 & 0.397 & 0.333 & 0.132 & 0 & -0.667 \\ 0 & 0 & 0 & 0 & 1 & 0.202 & 0 & 0.067 & 0 & 0 \\ 0 & 0 & 0 & 0 & 0 & 1 & 0 & 0.333 & 0 & 0\end{array}\right]\left[\begin{array}{c}p_{8} \\ p_{4} \\ p_{7} \\ p_{6} \\ p_{3} \\ p_{2} \\ p_{5} \\ p_{1} \\ M_{j} \\ M_{k}\end{array}\right]=\left[\begin{array}{c}-1.898 q \\ -2.058 q \\ -1.306 q \\ -7.449 q \\ -1.075 q \\ -5.333 q\end{array}\right]$

Equation 13 can be partitioned into a square matrix relating to the first six unknowns $\left(p_{8} \ldots p_{2}\right)$, and other terms relating to the remaining unknowns $\left(p_{5} \ldots M_{k}\right)$. The solution of the square matrix gives an equilibrium state, and the remaining four variables are introduced via the states of self-stress. Here, there are six equations in ten unknowns so the solution will involve four unknown values $\lambda_{i}$, each of which is a multiplier for one state of self-stress. After reordering the variables back to the natural order, the result is:

$\left[\begin{array}{c}p_{1} \\ p_{2} \\ p_{3} \\ p_{4} \\ p_{5} \\ p_{6} \\ p_{7} \\ p_{8} \\ M_{j} \\ M_{k}\end{array}\right]=\left[\begin{array}{c}0 \\ -5.333 q \\ 0 \\ 0 \\ 0 \\ -5.333 q \\ 0 \\ 0 \\ 0 \\ 0\end{array}\right]+\left[\begin{array}{cccc}0 & 1 & 0 & 0 \\ 0 & -0.333 & 0 & 0 \\ 0 & 0 & 0 & 0 \\ 0 & 0 & 0 & 0 \\ 1 & 0 & 0 & 0 \\ -0.333 & 0 & 0 & 0.667 \\ 0 & 0 & 0 & 0 \\ 0 & 0 & 0 & 0 \\ 0 & 0 & 1 & 0 \\ 0 & 0 & 0 & 1\end{array}\right]\left[\begin{array}{c}\lambda_{1} \\ \lambda_{2} \\ \lambda_{3} \\ \lambda_{4}\end{array}\right]$

Or

$$
\mathbf{p}^{*}=\mathbf{e}+\mathbf{S} \lambda
$$

It is worth considering the physical meaning of this equation.

- There is no load on the transverse strips $c-h$ and $d-g$, as a free-free beam with two regions cannot satisfy both moment and force equilibrium unless both regions carry no load; $p_{3}, p_{4}, p_{7}$ and $p_{8}$ must always be zero. If this had been recognised before the equations were set up, four variables could have been eliminated, as would four equations. The result would still have needed four states of self-stress.

- The internal forces on regions 2 and 6 are used to balance the forces applied on the remaining regions for the longitudinal strips - in other words the entire load is applied to the longitudinal strips, and these are balanced by forces from the transverse strip b-i. Strip a-j carries no load in the initial equilibrium position as defined by the e vector.

- $\lambda_{1}$ and $\lambda_{2}$ are multipliers for the first two states of self-stress, as defined by the first two columns of $\mathbf{S}$. Each refers to a state of self-stress in one of the longitudinal strips which allows the load to be shifted between strip a-j and strip b-i .

- The third column of $\mathbf{S}$ shows that the edge moment $M_{j}$ is entirely independent of the applied loads and can take any value, defined by $\lambda_{3}$, without nullifying the equilibrium of the system.

- The fourth state of self-stress, as defined by the final column of the $\mathbf{S}$ matrix, shows that any change to the edge moment $M_{k}$ is balanced by a change in the internal load $p_{6}$. An increase in $M_{k}$ corresponds to a decrease in the load on strip b-i . 
In more complex cases the physical interpretation of the states of self-stress is less obvious, and if the equations had been reordered differently, other variables might have been involved in the equilibrium state.

\section{OPTIMISATION}

The problem has now been reduced to its simplest form. Any combination of the various states of self-stress, as defined by the values of $\lambda_{i}$, will satisfy equilibrium (and all possible equilibrium states can be expressed by Eq. 14). The moments anywhere in the strips can be calculated in the normal way and compared with the moment capacity in each strip. The multiplier $Q$ that can be applied to all the loads $q_{i}$ without exceeding the moment capacity for any combination of $\lambda_{i}$ can thus be found. By definition, that value of $Q$ will be a lower bound on the collapse load of the slab.

To be useful, the highest lower bound has to be found. There are many optimisation techniques that have been devised, and a widespread literature. Press et. al. [8] give a good discussion of the techniques and pitfalls that can arise.

The Simplex algorithm (or a more sophisticated version thereof) is frequently used in cases where the limiting conditions can be expressed as simple functions, because it can lead directly to the optimal solution, but it is not suitable here because yield could occur at any point along a strip. It is thus very difficult to find simple expressions for bounds on the values of $\lambda_{i}$.

An alternative is Powell's method, which is sometimes referred-to as the Hill Climbing method. The technique conducts a series of one-dimensional optimisations along various vectors; the states of selfstress are a convenient set of vectors that have already been defined. By starting from the equilibrium state (defined by the vector $\mathbf{e}$ ) a search is made along one of the directions to find the value of $\lambda$ that gives the largest failure load. The process is repeated by moving along another search vector. If the function is smooth the procedure will zigzag its way to a (possibly local) maximum of the function and is reasonably efficient and simple to program.

When attempts were made to apply the technique to simple slab problems, the solution reached different finishing points depending on how the problem was set up. A simple example shows why. Figure 5 shows a simply supported slab divided into two longitudinal strips and four transverse strips under a uniformly distributed load. By enforcing the two lines of symmetry the problem can be reduced to two variables, which allows the value of $Q$ to be visualised for all combinations of $p_{i}$.

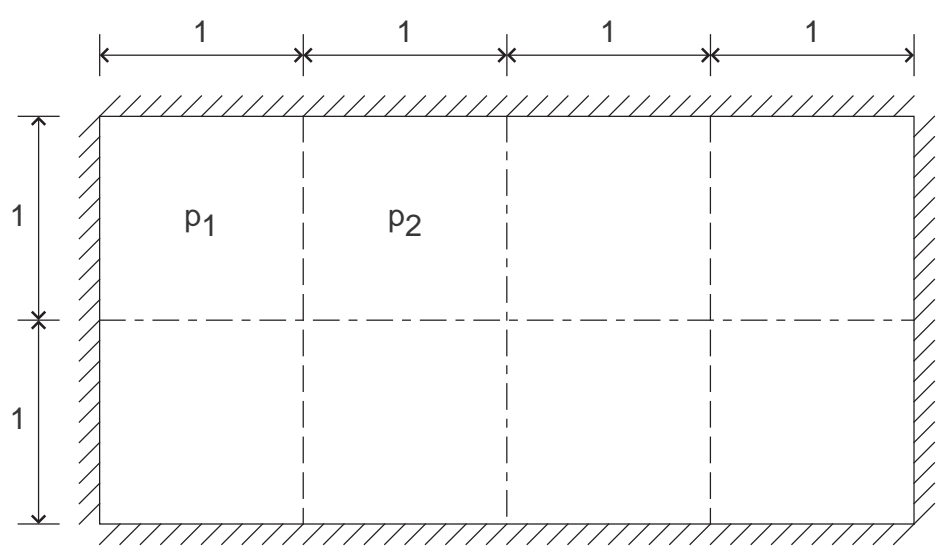

Fig 5. Two Degree of freedom problem

The maximum distributed load that the slab can carry for any value of $p_{1}$ and $p_{2}$ is plotted in Fig. 6 . The circles correspond to results where the numerical procedure stopped before reaching the highest point. The surface consists of a series of smooth regions, separated by discontinuities in slope. The finishing points of the optimisation, from different starting points, all lie on one or other of the ridge lines. An analogy explains why. Imagine a climber going up a mountain in fog who is constrained to walk only in N-S or E-W directions. If he reaches a point where any step takes him downhill, he will conclude he has reached the summit. But if he is on a ridge, running from NE-SW, he may be able to go higher, but he doesn't realise it. 


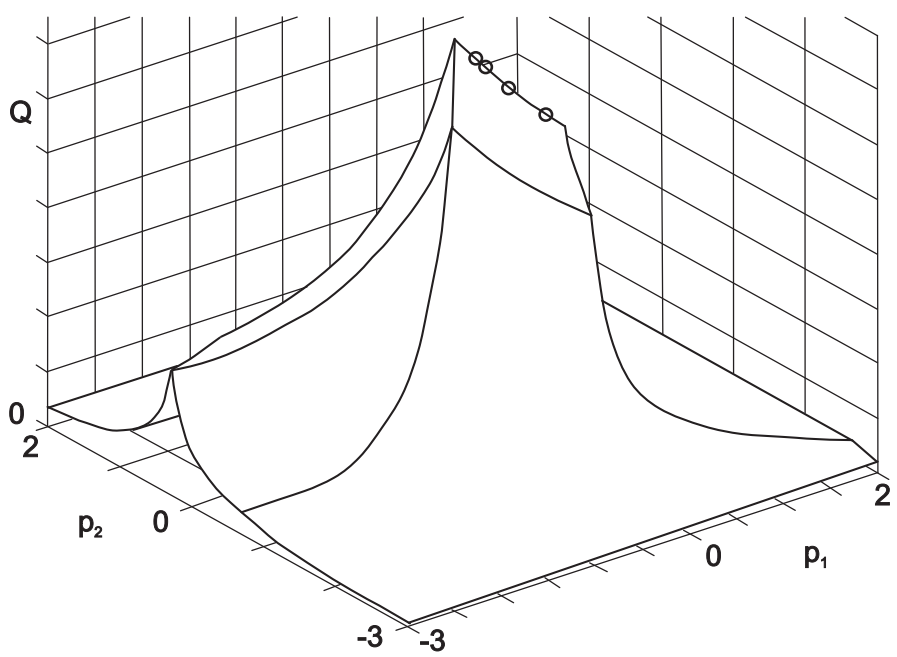

Fig 6. Slab capacity against $p_{1}$ and $p_{2}$.

Figure 7 shows the same surface as a contour plot. Each of the smooth regions corresponds to yield being reached in some, but not all, of the strips. Slope discontinuities occur where these surfaces intersect, and some of these discontinuities form ridges. The highest point is reached when several of the ridge lines meet, so to reach the summit the climber has to proceed along the ridge lines. If the iteration technique does not allow movement in these directions, the analysis will stop prematurely. Note that this is not the normal problem associated with reaching a local maximum; stopping on a ridge can occur even if the function has only a single global maximum.

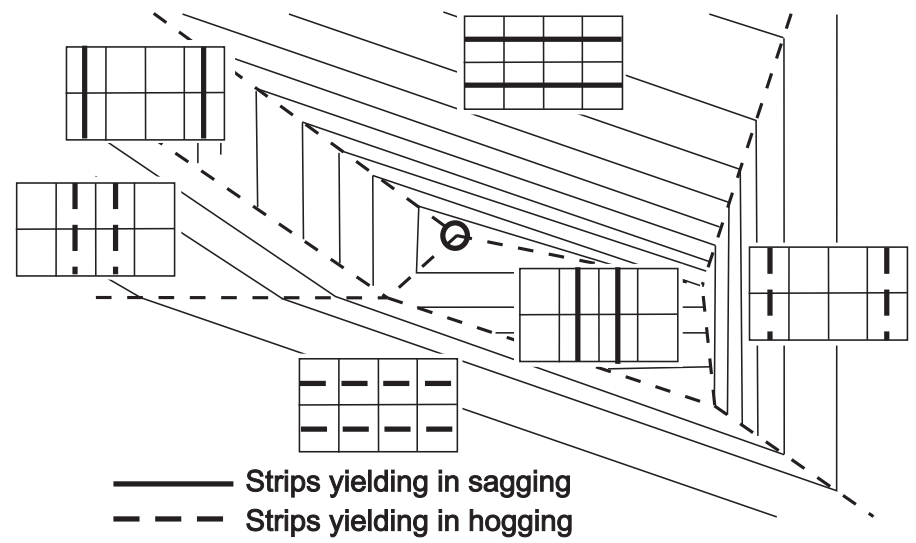

Fig 7. Contour plot of the 2-dimensional surface showing yielded Strips

(Some contours omitted for clarity).

To counteract this effect a modification has been made to the procedure. When the optimisation routine has reached its best estimate of the solution, a jump away is made by randomly choosing a small $\lambda$ vector to get a revised starting point. The optimisation is repeated and it is likely that the new optimum will also be on the same ridge. The line joining the two estimates of the optimum will be on, or near, the ridge line and can be added to the list of search vectors. In the climbing analogy the climber would be allowed to walk along the ridge.

In more realistic problems, there will be more variables, so it is less easy to visualise the process, but the optimum solution will still be the one where the largest possible number of strips are yielding, even if it is not always possible for all the strips to yield, and it is still possible to get stuck on a ridge line.

The whole procedure has been written in a computer program in $\mathrm{C}++$. The software is capable of analysing rectangular slabs, subdivided into rectangular regions with moment capacities specified in the same directions as the strips, and can take account of free, fixed and simply-supported edges, slabs that contain holes, column supports and lines of symmetry. Extensions to other shapes, and 
more complex reinforcement arrangements, would be straightforward but have not been implemented here.

\section{EXAMPLES}

\subsection{Example 1 - slab designed by strip theory.}

Figure 8 shows a simply supported slab, of aspect ratio 1.5. Reynolds [9] gives a strip design procedure for such a slab, subject to a uniformly distributed load, which leads to the required moment capacities in sagging bending as shown in Fig. 8(a). These moment capacities were used as the input for the analysis procedure given here, which, after a few seconds, leads to the distribution of loads as shown in Fig. 8(b). This is precisely the distribution suggested by Reynolds, and this example represents the sort of problem likely to be faced by the modern assessor.

This example shows that the technique is capable of recovering the assumptions that were made by the original designer. It is the inability to do that with a finite element program that leads to structures being repaired unnecessarily, since the checker is unable to find the load distribution that the original designer found to be adequate. If the original design was competent before, this method should show that the structure remains competent. It cannot, however, "turn a sow's ear into a silk purse"; if the original design was inadequate, or the design load has changed significantly, or the structure has deteriorated with time, it may still fail.

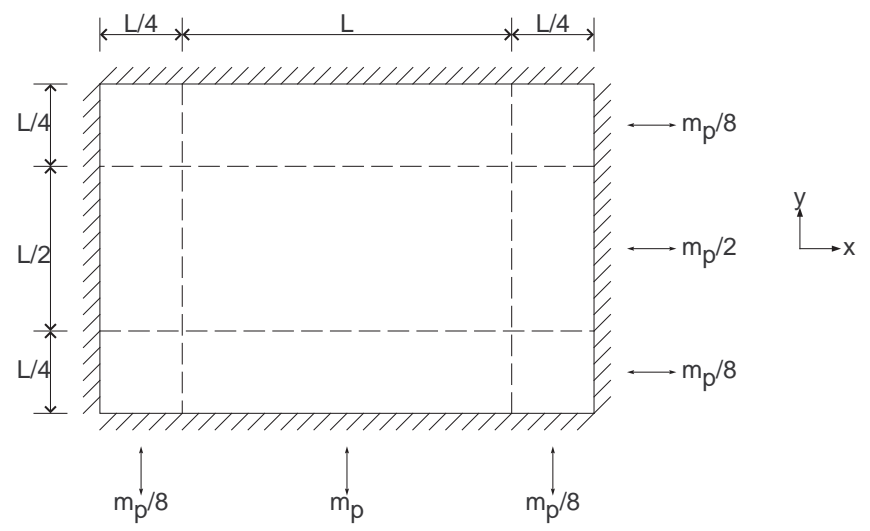

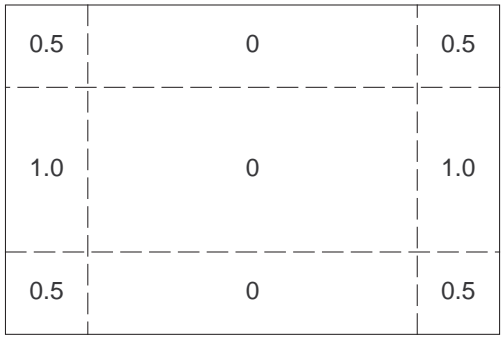

Proportion of load applied to $\mathrm{x}$-direction strips

(b)

Fig 8. Layout of slab used in Example 1.

(a) shows the input moment capacities

(b) shows the load proportions determined by the analysis

The method has been checked against other design cases, with similar results. In practice, the reinforcement detailer would usually have made conservative assumptions of capacity, so in many cases it is found that the structure can carry more load than the original designer intended.

It would be possible to stop at this point and be satisfied with a tool that can verify existing good design, and indeed a result such as this could have been achieved, albeit by a different route, using O'Dwyer and O'Brien's [7] technique. There are however other problems that need to be tackled when assessing existing structures, which show that the method, as explained so far, has limitations.

\subsection{Example 2. Short Single Span with Concentrated Load}

The second example is of point loads applied to a simply-supported, single-span bridge deck. Many such structures are currently being reassessed due to the requirements for heavier truck loads. The predicted failure patterns for such structures involve partial width yield line patterns with fans. Several models of such slabs were tested by Collins [10]. Two of the slabs had limited amounts of reinforcement, but one slab, No. 3, had top and bottom steel in both directions. 


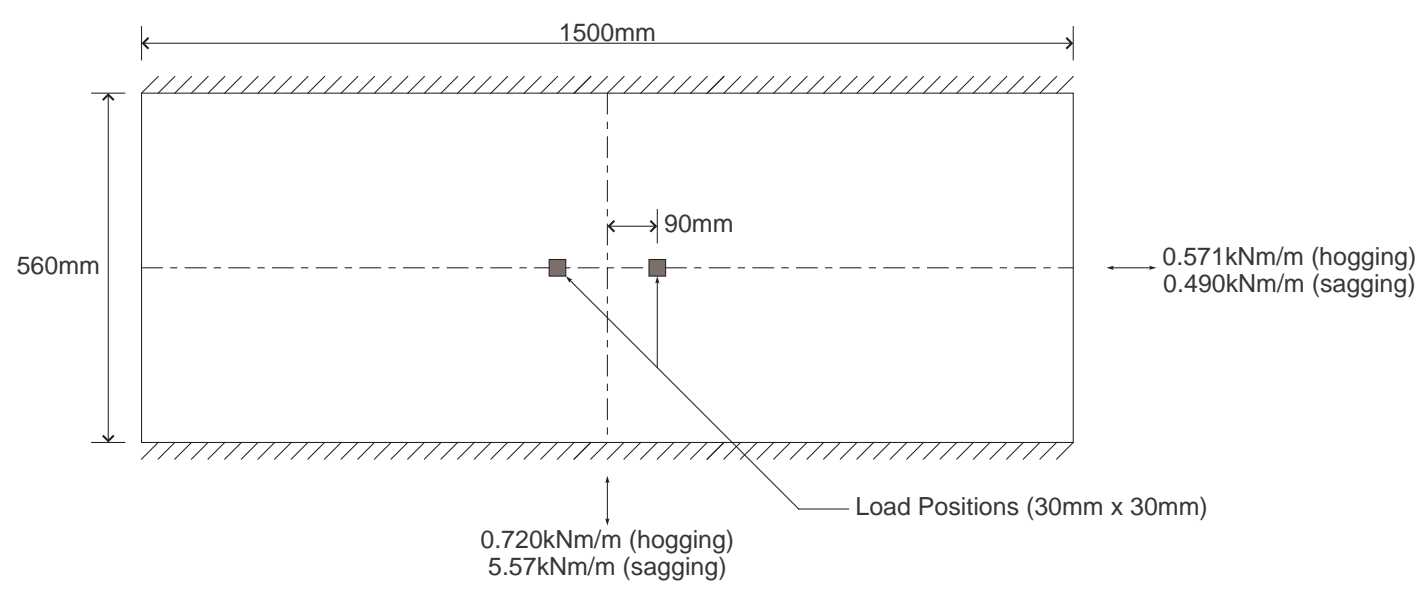

Fig 9. Collins's slab 3.

The slab layout is shown in Fig. 9; two point loads were applied symmetrically on $30 \mathrm{~mm}$ square pads as shown. The maximum load carried by the slab was $31.3 \mathrm{kN}$ (shared between the two loading points), and the final crack pattern on the underside is shown in Fig. 10. Collins carried out yield line analyses of the slab and obtained the lowest upper bound of $25.6 \mathrm{kN}$ for the mechanism shown in Fig. 11. She attributed the fact that the slab carried more than its upper-bound capacity to strainhardening in the steel and the development of membrane actions which are known to be important for point loads applied to slabs where compressive arching action can be resisted by ring tension in the reinforcement away from the loading positions.

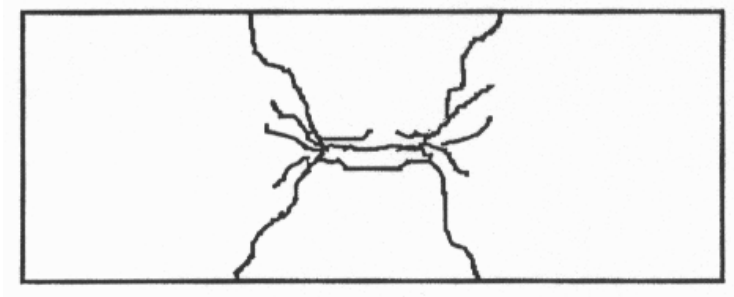

Fig 10. Final crack pattern on underside of Collins's slab

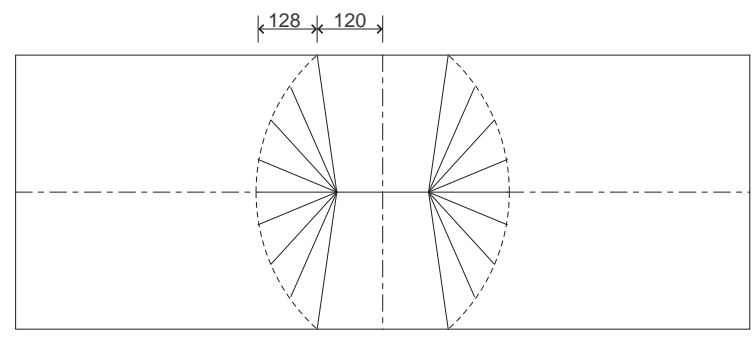

Fig 11. Yield line pattern

\subsection{Lower-bound Analysis}

The slab has been re-analysed using the methods described in this paper. To minimise the number of variables, one quarter of the slab was analysed, using the strip layout shown in Fig. 12. The loading patch was increased in size by adding one half of the slab thickness all round to allow for local load spreading. The values of the moment capacity determined by Collins from material tests were used.

The program converged very slowly to a solution, and the highest lower bound found was $17.6 \mathrm{kN}$, some $33 \%$ lower than the lowest upper bound. Figure 13 shows the moment distributions in the various strips. The dominance of the sagging bending in the transverse direction under the load is clear, as is the absence of moment in the right hand half of the slab. But in the area in between, where the fan mechanism developed, and as predicted by the yield line method, the situation is far from simple, and no clear pattern can be discerned.

It is clear that the method, as presently constituted, is not good at determining when yielding takes place at an angle to the line of action of the strip. To see why that might occur, a case is needed for which good upper and lower bounds are known. 


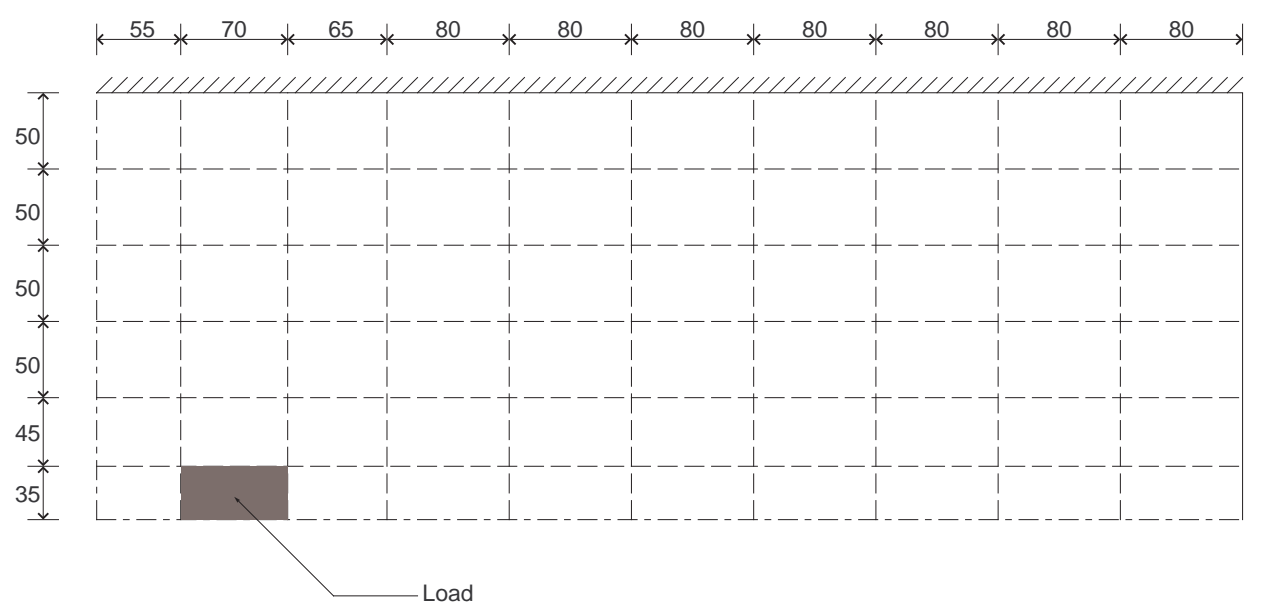

Fig 12. Strip layout used to analyse Collins slab (dimensions in $\mathrm{mm}$ )

\section{Longitudinal Strips}

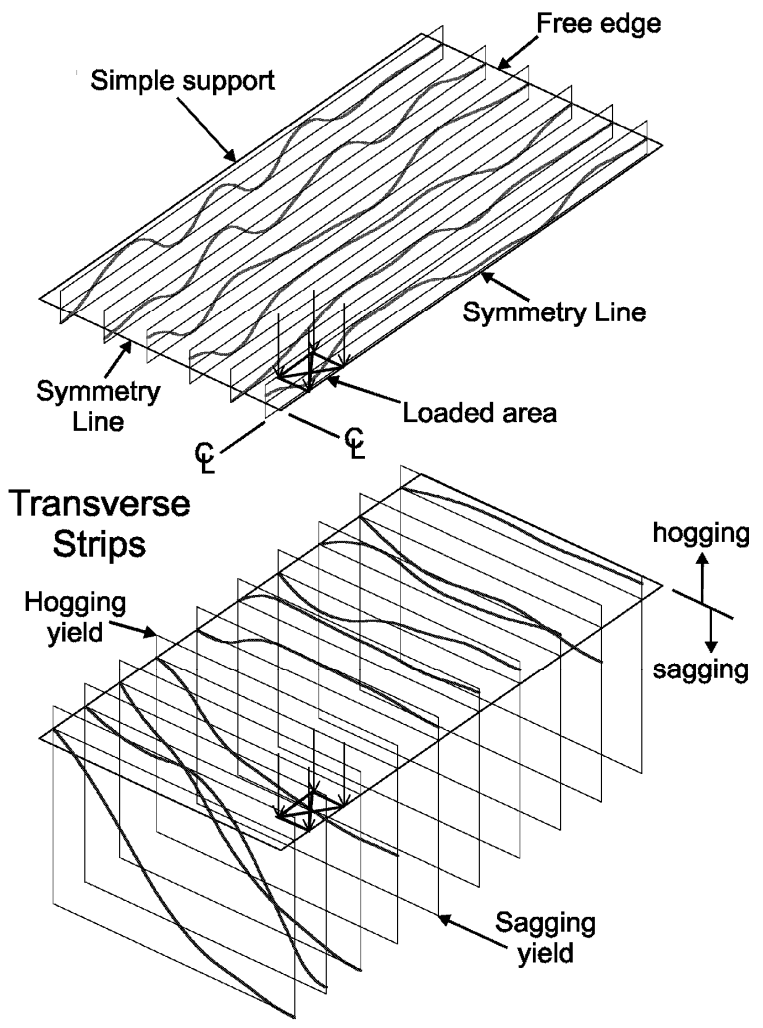

Fig 13. Moment distributions in strip analysis of Collins's slab. Upper view shows strips parallel to the support - lower view shows strips between supports.

Only one quarter of the slab is shown for clarity.

\subsection{Example 3 - Fully Fixed Square Slab}

One of the few non-trivial cases for which identical upper- and lower-bounds are known is the clamped square slab (Fig. 14), which was analysed by Fox [11]. Both the distribution of moments for his lower-bound, and his upper-bound collapse mechanism, are necessarily complex, involving in the one case parabolic and hyperbolic moment distributions in the corners and in the other complex corner fans. The details are of no concern here, but his result is important. He showed that the true 
collapse load of the slab $Q$ was given by $Q L^{2} / m_{p}=42.85$, where $L$ is the span and $m_{p}$ is the moment capacity per unit width in both sagging and hogging bending.

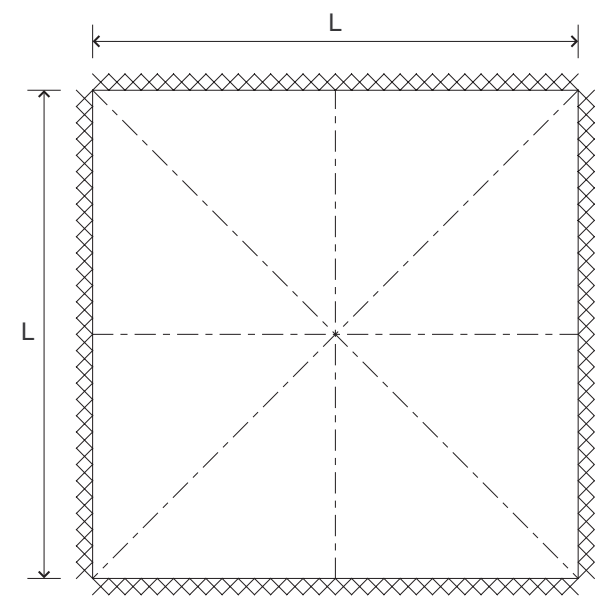

Fig 14. Fully-fixed square slab under uniformly distributed load, showing 8-fold symmetry

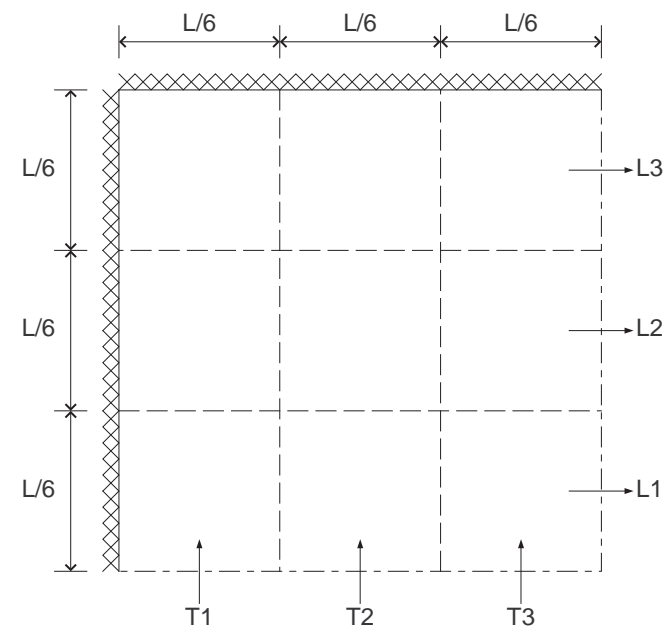

Fig 15. Input to Lower Bound Analysis for Example 3 Showing Strip Divisions.

\subsection{Lower-bound Analysis Results}

Figure 15 shows the layout used for the lower bound analysis, which was performed on a slab nominally $10 \mathrm{~m}$ square. Two lines of symmetry are enforced, and the resulting $5 \mathrm{~m} \times 5 \mathrm{~m}$ slab divided into 3 strips in each direction.

The lower bound analysis predicts a maximum load of $Q L^{2} / m_{p}=32$, but there are several different arrangements of $\lambda_{i}$ that generate the same maximum. All the strips are at yield in hogging at the supports and in sagging at midspan, although the bending moment distributions differ between strips as well as between the different $\lambda_{i}$ distributions. This reflects the fact that there is no advantage in altering the moment distribution away from the yielding region; a similar phenomenon was observed in the previous example in the unloaded half of the slab.

The analysis has found the solution corresponding to a uniformly distributed load $w$ applied to a fixed-ended beam with moment capacity $m_{p}$, which would give a collapse load of $w=16 m_{p} / L^{2}$. In the present case, half the load goes in each direction giving $w=32 m_{p} / L^{2}$.

It is possible that the routine has found a local maximum for $Q$, and that a higher global maximum exists but this cannot be checked without an exhaustive search, which would be prohibitively costly in computer time. Doubling (or even quadrupling) the number of strips in each direction does not alter the solution that is found.

Consideration of the failure patterns shows why this might be the case. Figure 16 shows the "yield line pattern" that would be generated by the lower bound that is predicted here. Because the lower bound does not correspond to the true collapse load this pattern does not satisfy the compatibility conditions. Figure 17 shows the classic yield line pattern for a clamped slab that does satisfy compatibility. It consists of diagonal sagging lines with corner fans. Even without the fans, this gives an upper bound of $Q L^{2} / m_{p}=48$ which is much closer to the true value than the lower bound produced here. It is immediately obvious that the two patterns differ widely. It is also obvious that a strip analysis, with strips running horizontally and vertically, will not have strips perpendicular to most of the yield lines, so cannot be expected to have a stress field that is close to that which follows from the yield line pattern. It is thus unsurprising that the lower bound obtained by the strip method differs from the true solution.

The slab has also been analysed using strips parallel to the diagonals, as shown in Fig. 18. If load is shared equally between the two sets of strips, then the longest diagonal will govern and since it is $\sqrt{ } 2$ longer than the side, the lower bound load will be halved to $Q L^{2} / m_{p}=16$. This can be improved if load is shared between the strips in a way that is linearly (although not proportionally) related to the relative stiffnesses of the two strips that cross at any point. Load can then be shed from long strips to short strips in the corners. The highest lower bound that has been found this way is $Q L^{2} / m_{p}=25.13$. This is well below the collapse load predicted by having strips parallel to the sides. 


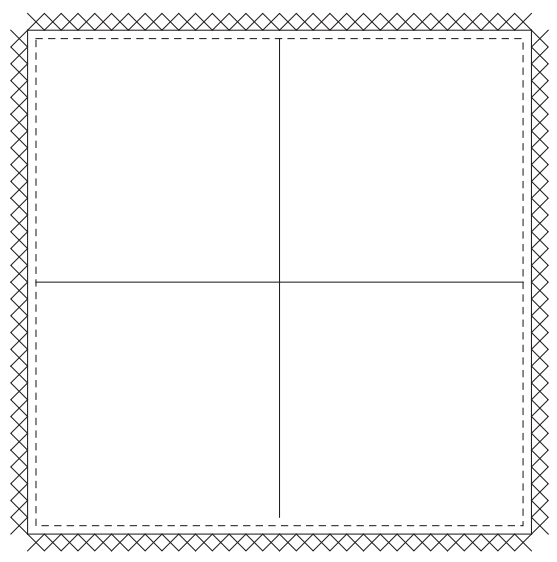

Fig 16. Incompatible "Yield Line pattern" predicted by Lower Bound Analysis.

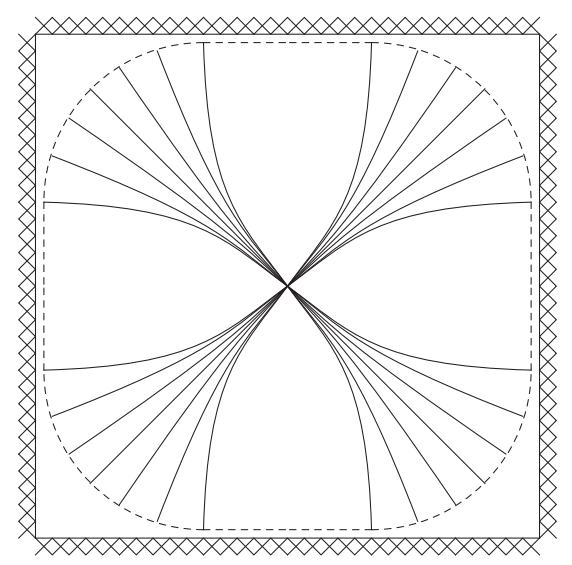

Fig 17. Corner fan yield line pattern

Analogous effects have been noted before, but usually in connection with point loads, such as those imparted to flat slabs by supporting columns. Hillerborg [12] himself recognised the problem and introduced the idea of a rectangular corner-supported element which distributed the concentrated forces from the column to conventional strips elsewhere.

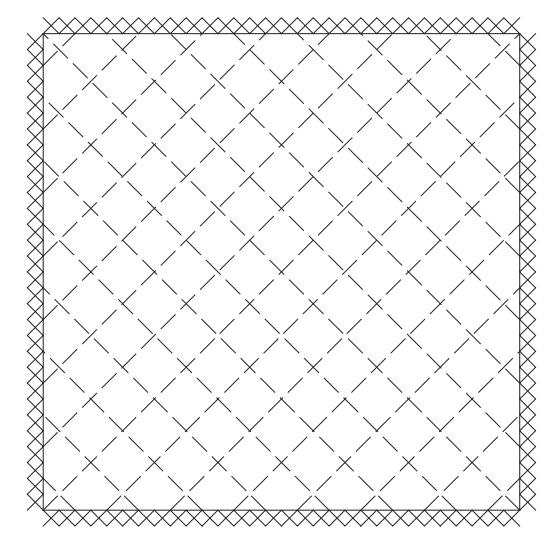

Fig 18. Diagonal strips

Rozvany [13] and Morley [14] introduced discontinuities in the moment field in two orthogonal directions, which allow the loads to be carried by diagonal shear forces towards the concentrated load. This overcomes the problem that axisymmetric moment patterns around a point load necessarily involve torsion, which is not allowed in Hillerborg strips. Rozvany also showed that the optimal design of slabs with corners required the use of diagonal strips spanning across the corners.

All of this work, and similar studies that followed, were concerned with the design problem, and have not been widely used in practice. Satisfactory designs can be produced without worrying about optimality conditions, and finite element methods, combined with the use of the Wood-Armer [15] equations, lead to structures that can safely carry the desired loads. But that is not the problem that is being addressed here where a good lower-bound analysis technique is required. The fundamental problem is that the strip method is poor at distributing loads in directions that run at an angle to both sets of strips.

\section{ANALYSIS WITH FOUR SETS OF STRIPS}

One way of introducing $M_{x y}$ moments is to add strips at $\pm 45^{\circ}$ to the original strips. The applied load can still be applied to one set of strips, as has been done in the previous analysis, but instead of one set of self-equilibrating inter-strip forces ( $p_{i}$ above) it will now be necessary to have three.

The question of the moment capacity has to be addressed. In the Hillerborg strip analysis it is assumed that there are two layers of steel, each of which contributes to the moment capacity in one 
set of strips; interactions between the strips can be conveniently ignored. With four sets of strips that will no longer be the case. For any given combination of the expanded set of inter-strip forces $p_{i}$ it will be necessary to determine the corresponding moment triad $\left(M_{x}, M_{y}, M_{x y}\right)$ at any point of interest. This can then be compared with a suitable yield function so that the safe load multiplier can be determined.

To determine the $\left(M_{x}, M_{y}, M_{x y}\right)$ values at one point the values from four strips have to be combined. Figure 19 shows how the moments from 4 strips should be combined. $a-a$ and $b-b$ are parallel to the $x$ - and $y$ - axes respectively, while $u-u$ and $v-v$ are at $45^{\circ}$. Fig. 19(b) shows how the moments in the $45^{\circ}$ elements are rotated to the $0-90^{\circ}$ direction using Mohr's circle. To these must be added the moments from $a-a$ and $b-b$ to give the total moment field, which must be compared with the moment capacity described above. The resulting equations are:

$$
\begin{aligned}
& M_{x}=M_{a}+\frac{\left(M_{v}+M_{u}\right)}{2} \\
& M_{x y}=\frac{\left(M_{v}-M_{u}\right)}{2} \\
& M_{x}=M_{b}+\frac{\left(M_{v}+M_{u}\right)}{2}
\end{aligned}
$$
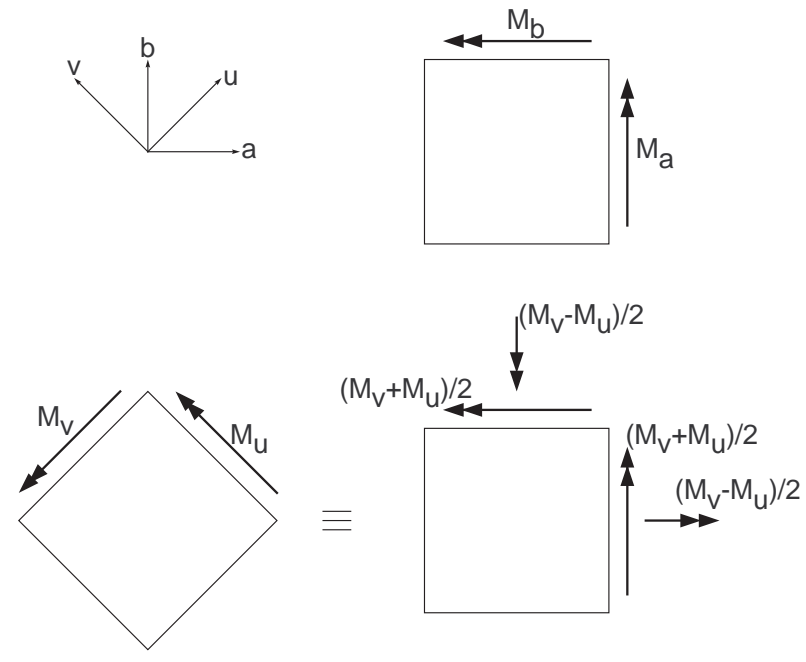

Fig 19. Combining moments from 4 strips.

The method can be applied to any suitable yield function. Denton and Burgoyne [16] presented a method of assessing the reserve moment capacity of a slab subjected to a given set of moments $\left(M_{x}\right.$, $\left.M_{y}, M_{x y}\right)$. That method differs from the Wood-Armer equations normally built into computer programs, which are intended for design of slabs and which include an optimality condition that is not required when performing an analysis. However, any appropriate yield function could be used. For example, May et al [17] showed that, in certain circumstances, especially when $M_{x y}$ moments were high, the Johanson yield criterion was unconservative. Because the method presented here checks the yield state at all points, it is possible to check whether May's yield condition should be substituted for the normal condition at any particular point. It would also be possible to use an llyushin [18] or von-Mises yield surface for analysing steel structures.

It should be noted that this analysis with four sets of strips only satisfies the moment capacity conditions at discrete points, which need to be predefined. When the moment capacities of two strips are independent, as they are when only two orthogonal strips are used, the capacity can be checked at any point, but when four strips are used, various complications arise. The moment capacity has to be checked at locations where the moments in all four strips are known. The diagonal strips are not perpendicular to the supports, so the length of each strip varies. The refined method thus has to be applied to a regular square grid so that moments in each strip are calculated at the same point. It also 
means that a more accurate solution will be obtained by increasing the number of strips, at the expense of significantly increased computation time.

A revised version of the program has been written for the particular case of a square clamped plate under u.d. load, and uses the Denton and Burgoyne approach, so the results should be directly comparable with Fox's exact solution and normal yield-line analyses. The program exploits the various symmetries in the slab so that only one quarter has to be analysed, but even so there are far more variables than when only two orthogonal strips are used, and each calculation is more complicated. Nevertheless, by dividing one quarter of a fixed plate into a $6 \times 6$ grid, the highest lower bound found is $Q L^{2} / m_{p}=41.19$, which is much closer to Fox's value than that obtained with only two sets of strips. It is possible that this is not the highest lower bound that could be achieved with this layout of strips, but it does represent a valid lower bound on the collapse load of the slab.

Figure 20 shows the nodal locations where the slab is yielding at the highest lower bound found. Although directions of yielding are not shown, the pattern is remarkably similar to the exact form found by Fox.

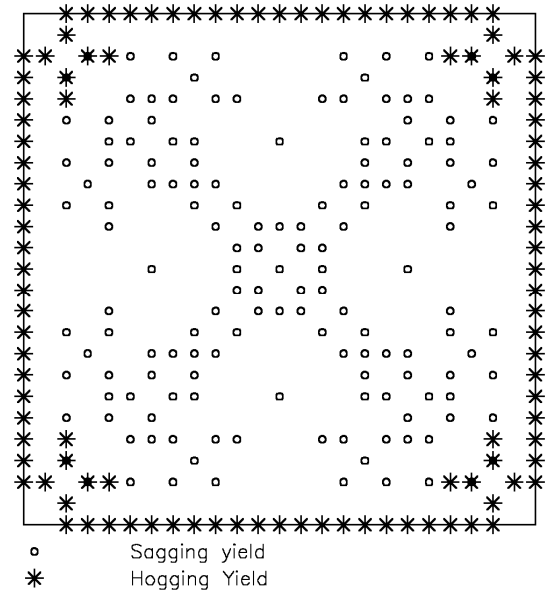

Fig 20. Yielding locations in highest lower bound

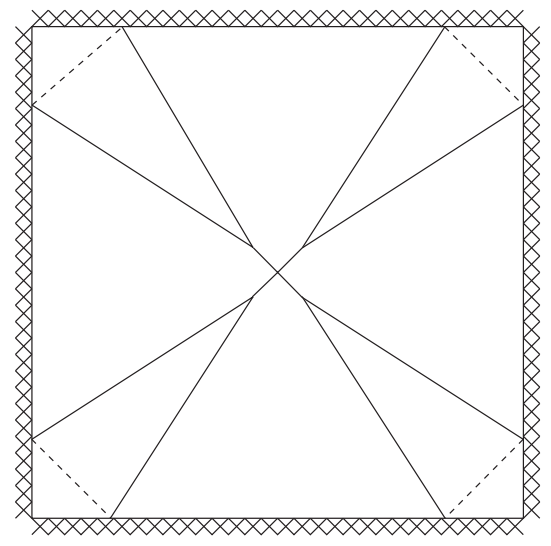

Fig 21. Predicted Yield Line Pattern for Example 3 from COBRAS [6].

\section{OTHER ANALYSES OF SQUARE SLAB}

There have been many other analyses of the square slab problem, several of which were summarised by Fox[11]. Wood's analysis[19] gave $Q L^{2} / m_{p}$ as 43.852; Mansfield [20] gave 42.895 and an improved version by Morley [21] gave 42.880, and although these patterns are simpler than that of Fox, they are too complicated for routine use. Analysis using an automated yield line program (COBRAS) [6] gives an upper bound of $Q L^{2} / m_{p}=43.99$, using the pattern shown in Fig 21.

\subsection{Finite Element Analysis}

An analysis of a square slab has also been carried out using a nonlinear finite element program (Abaqus). This requires specific dimensions and material properties, and will also model membrane effects, so the results will not be exactly comparable to the other results quoted. The slab was modelled as a homogeneous material with an elastic modulus and moment capacity equivalent to a concrete slab with a single layer of reinforcement at mid-depth ( $\left.E=28 \mathrm{GPa}, m_{p}=20 \mathrm{kNm}\right)$. The analysis of the slab using a fully non-linear procedure gave an ultimate collapse load of $Q L^{2} / m_{p}=47.63$, at which point both concrete crushing and steel yielding are taking place. This load is higher than the loads given by the upper bound solution, but is enhanced by the membrane action which both upperand lower-bound methods ignore. If analysed using linear finite elements, as would normally be undertaken for assessment, the steel starts to yield at $Q L^{2} / m_{p}=6.84$, which shows how uninformative such programs can be. The load-deflection curve for the finite element analysis is shown in Fig. 22, with the other results shown for comparison. 


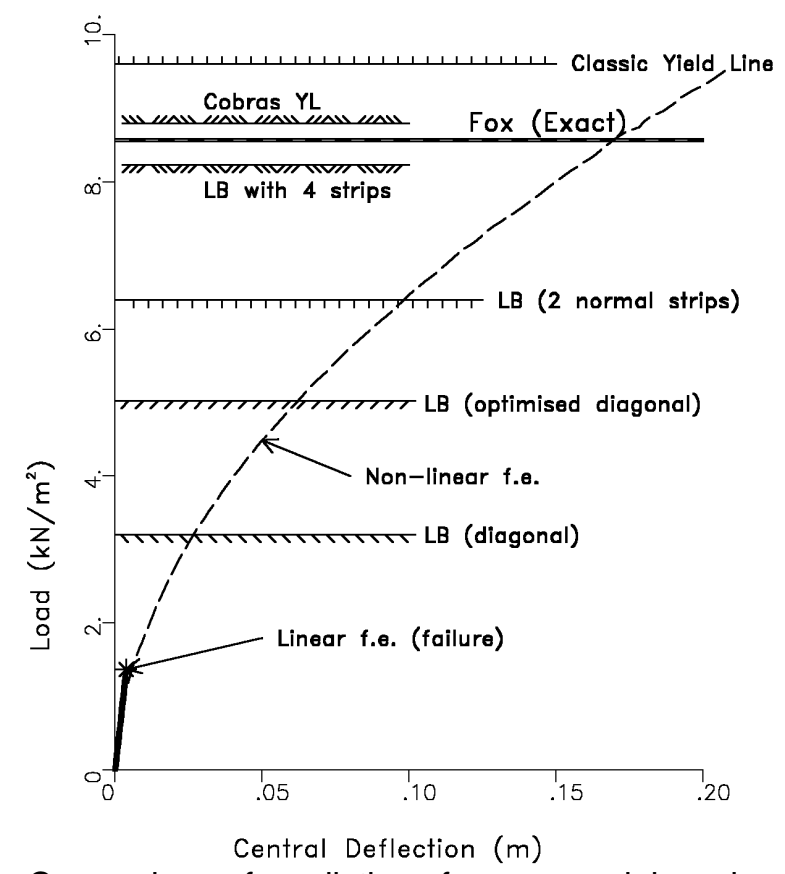

Fig 22. Comparison of predictions for square slab under u.d.load

\section{DISCUSSION}

The results of all the different analyses and theories are shown in Table 1. As expected the upper and lower bounds bracket the exact solution. The COBRAS result is $3 \%$ high, and the Lower Bound method is $4 \%$ low. In real problems the exact solution is not known, so this method offers the real possibility of giving the checker some bounds on the degree of uncertainty about the slab's capacity.

The optimisation method used for this analysis is the same as that utilised when there are only two sets of strips, but the method converges very slowly, and it is by no means clear that the true optimum has been reached. Hill-climbing techniques are greedy, in the sense that they walk as fast as possible up the nearest hill, and are likely to get stuck on a local maximum, or at a point from which they cannot escape. For more complex problems it would probably better be better to use an alternative technique, such as simulated annealing [8], which has been used to solve the notorious "travelling salesman" problem. This technique starts by allowing quite large random jumps in the various parameters (here the $p_{i}$ values), and then slowly reducing the jump size as the solution is approached.

Table 1: Values for $\mathrm{QL}^{2} / \mathrm{m}_{\mathrm{p}}$ using various analysis methods.

\begin{tabular}{|l|c|c|}
\hline \multicolumn{1}{|c|}{ Method } & $Q L^{2} / m_{p}$ & Factor \\
\hline Linear Elastic Finite Element & 6.84 & 0.160 \\
\hline Lower Bound with simple diagonal strips & 16 & 0.373 \\
\hline Lower Bound with optimised diagonal strips & 25.13 & 0.586 \\
\hline Lower Bound with two strips parallel to sides & 32 & 0.747 \\
\hline Lower Bound with four strips & 41.19 & 0.961 \\
\hline Fox's Exact Solution [11] & $\mathbf{4 2 . 8 5}$ & $\mathbf{1}$ \\
\hline Best Yield Line - Mansfield \& Morley & 42.88 & 1.001 \\
\hline COBRAS Yield Line & 43.99 & 1.026 \\
\hline Fully Plastic Finite Element & 47.63 & 1.112 \\
\hline Classic Yield Line Analysis & 48 & 1.120 \\
\hline
\end{tabular}




\section{CONCLUSIONS}

This study has shown:-

1. It is possible to construct an automated procedure to analyse slabs using the Hillerborg Strip Method, which is normally used only for design.

2. It is necessary to include self-equilibrating inter-strip forces to allow load to be transferred into strips that are otherwise unloaded.

3. The load distribution within the slab can be expressed in terms of an equilibrium state and a set of states of self-stress. By varying the amount of each state of self-stress that is added, all valid combinations forces and moments that satisfy equilibrium can be described.

4. A valid lower bound can be found from each of these equilibrium states.

5. An automated procedure can be used to find the highest lower-bound, but this must include a procedure to prevent the solution stopping at a non-optimal position.

6. The method can be used to recover the assumptions made in the original design, even if those calculations are no longer available, which allows the method to be used for assessment of existing structures.

7. Comparison with published values shows that lower bounds can be calculated, but they may be well below the true collapse load of the structure. This is because the $M_{x y}$ moments are being ignored.

8. By adding two additional sets of strips at $\pm 45^{\circ}$ a higher lower bound can be obtained which is much closer to the true collapse load.

9. More care is needed when combining together the applied moments in four sets of strips since the moment capacities are no longer independent.

10. The method is not restricted to one yield function.

\section{References}

[1] Burgoyne, C.J., Are structures being repaired unnecessarily?, The Structural Engineer, 82/1, 22-26, 2004.

[2] Hillerborg, A., Jämviksteori för armerade betongplatter, (Equilibrium theory for Concrete Slabs), Betong, 41/4, 171-182, 1956.

[3] Hillerborg, A., Strip Method of Design, Spon Press, 1976.

[4] Calladine C.R., Plasticity for Engineers, Theory and Applications, Horwood, 2000.

[5] May I.M. and Mann A. Personal Communication

[6] Middleton, C.R., Concrete bridge assessment: an alternative approach, Structural Engineer, 75/23, 403-409, 1977.

[7] O'Dwyer, D.W. and O'Brien, E.J.: Design and analysis of concrete slabs using a modified strip method', Struct. Eng., 76/17, 329-333, 1998.

[8] Press, W.H., Teukolsky, S.A., Vetterling, W.T. and Flannery, B.P.: Numerical Recipes in C++, The Art of Scientific Computing, Second Edition, Cambridge University Press. 2002.

[9] Reynolds C.E. and Steedman J.C., Reinforced Concrete Designers Handbook, $9^{\text {th }}$ Edition, Viewpoint, Slough, 1981.

[10] E.Collins E., Strength Assessment of Concrete Bridge Slabs with Low Transverse Reinforcement, MEng Thesis, University of Cambridge, 1997.

[11] Fox E.N., Limit Analysis for Plates: The Exact Solution for a Clamped Square Plate of Isotropic Homogeneous Material Obeying the Square Yield Criterion and loaded by uniform pressure, Proc. R. Soc. Lond., A277, 121-155, 1974.

[12] Hillerborg A., The Advanced Strip Method - a Simple Design Tool, Mag. Conc. Res., 34/121, 175-181, 1982

[13] Rozvany G.I.N., Optimal Design of Flexural Systems, Pergamon, 1976

[14] Morley C.T., Equilibrium Design Solutions for Torsionless Grillages or Hillerborg Slabs under Concentrated Loads, Proc. Inst. Civ. Engrs Part 2, 81, 447-460, 1986

[15] Wood R.H., The reinforcement of slabs in accordance with a predetermined field of moments, Concrete, 2, 69-76, 1968, and correspondence by Armer G.S.T, Concrete, 2, 319-320, 1968.

[16] Denton S.R. and Burgoyne C.J., The assessment of reinforced concrete slabs and bridge decks, The Structural Engineer, 74/9, 147-152, 1996. 
[17] May, I.M., Montague, P., Samad, A.A.A. Lodi, S.H., Fraser, A.S., The behaviour of reinforced concrete elements subject to bending and twisting moments, Procs Inst. Civ. Engs: Struct. and Build., 146/2, 161-171, 2001.

[18] Burgoyne C.J. and Brennan M.G., Exact Ilyushin Yield Surface, Int. J. Solids \& Structures, 30/8, 1113-1131, 1993

[19] Wood R.H., Plastic and Elastic Design of Slabs and Plates, Thames and Hudson, 1961

[20] Mansfield E.H., Studies in collapse analysis of rigid-plastic plates with square yield diagram, Proc. R. Soc. Lond. A241, 311-338, 1957

[21] Morley C.T., The Ultimate bending Strength of Reinforced Concrete Slabs, PhD Dissertation, University of Cambridge, 1965 